\title{
CONTROLLABILITY OF 3D INCOMPRESSIBLE EULER EQUATIONS BY A FINITE-DIMENSIONAL EXTERNAL FORCE
}

\author{
HAYK NERSISYAN ${ }^{1}$
}

\begin{abstract}
In this paper, we study the control system associated with the incompressible 3D Euler system. We show that the velocity field and pressure of the fluid are exactly controllable in projections by the same finite-dimensional control. Moreover, the velocity is approximately controllable. We also prove that 3D Euler system is not exactly controllable by a finite-dimensional external force.
\end{abstract}

Mathematics Subject Classification. 35Q35, 93C20.

Received October 21, 2008. Revised February 20, 2009.

Published online July 2nd, 2009.

\section{INTRODUCTION}

Let us consider the controlled incompressible 3D Euler system:

$$
\begin{aligned}
\dot{u}+\langle u, \nabla\rangle u+\nabla p & =h+\eta, \quad \operatorname{div} u=0, \\
u(0, x) & =u_{0}(x)
\end{aligned}
$$

where $u=\left(u_{1}, u_{2}, u_{3}\right)$ and $p$ are unknown velocity field and pressure of the fluid, $h$ is a given function, $u_{0}$ is an initial condition, $\eta$ is the control taking values in a finite-dimensional space $E$, and

$$
\langle u, \nabla\rangle v=\sum_{i=1}^{3} u_{i}(t, x) \frac{\partial}{\partial x_{i}} v .
$$

We assume that space variable $x=\left(x_{1}, x_{2}, x_{3}\right)$ belongs to the $3 \mathrm{D}$ torus $\mathbb{T}^{3}=\mathbb{R}^{3} / 2 \pi \mathbb{Z}^{3}$.

The question of global well-posedness of 3D Euler system continues to be one of the most challenging problems of fluid mechanics. However, the local existence of solutions is well known (e.g., see [15,16]). Moreover, Beale et al. [3] proved that under the condition

$$
\int_{0}^{T}\|\operatorname{rot} u(t)\|_{L^{\infty}} \mathrm{d} t<\infty
$$

the smooth solution exists up to time $T$.

Keywords and phrases. Controllability, 3D incompressible Euler equations, Agrachev-Sarychev method.

1 CNRS (UMR 8088), Département de Mathématiques, Université de Cergy-Pontoise, Site de Saint-Martin, 2 avenue Adolphe

Chauvin, 95302 Cergy-Pontoise Cedex, France. Hayk.Nersisyan@u-cergy.fr 
In this paper, we show that for an appropriate choice of $E$, the problem is exactly controllable in projections, i.e., for any finite-dimensional subspaces $F, G \subset H^{k}$ and for any $\hat{u} \in F, \hat{p} \in G$ there is an $E$-valued control $\eta$ such that problem (1.1), (1.2) has a solution $(u, p)$ on $[0, T]$ whose projection onto $F \times G$ coincides with $(\hat{u}, \hat{p})$ at time $T$. We also prove that the velocity $u$ is approximately controllable, i.e., $u(T)$ is arbitrarily close to $\hat{u}$. From equation (1.1) it follows that the pressure can be expressed in terms of the velocity, so we can not expect to control approximately the pressure and the velocity simultaneously. The proofs of these results are based on a development of some ideas from $[1,2,12,13]$.

Let us mention some earlier results on the controllability of the Euler and Navier-Stokes systems. The exact controllability of Euler and Navier-Stokes systems with control supported by a given domain was studied by Coron [5], Fursikov and Imanuvilov [8], Glass [9], and Fernández-Cara et al. [7]. Agrachev and Sarychev [1,2] were first to study controllability properties of some PDE's of fluid dynamics by finite-dimensional external force. They proved the controllability of 2D Navier-Stokes and 2D Euler equations. Rodrigues [11] used AgrachevSarychev method to prove controllability of 2D Navier-Stokes equation on the rectangle with Lions boundary condition. Later Shirikyan [13] generalized this method to the case of not well-posed equations. In particular, the controllability of 3D Navier-Stokes equation is proved.

Notice that the above papers concern the problem of controllability of the velocity. In this paper, we first develop the ideas of these works to get the controllability of the velocity of 3D Euler system. One of the main difficulties comes from the fact that the resolving operator of the system is not Lipschitz continues in the phase space. We next deduce the controllability of the pressure from that of the velocity with the help of an appropriate correction of the control function.

We also treat the question of exact controllability of 3D Euler equation. In [14], Shirikyan shows that the set of attainability $A_{T}\left(u_{0}\right)$ of $2 \mathrm{D}$ Euler equation from initial data $u_{0} \in C^{s}$ at time $T>0$ cannot contain a ball of $C^{s}$. We show that the ideas of [14] can be generalized to prove that the set $A\left(u_{0}\right)=\cup_{T} A_{T}\left(u_{0}\right)$ also does not contain a ball in 3D case. In particular, 3D Euler equation is not exactly controllable.

The paper is organized as follows. In Section 2, we give a perturbative result for 3D Euler system. In Sections 3 and 4, we formulate the main results of this paper, which are proved in Sections 5 and 6. Section 7 is devoted to the problem of exact controllability.

Notation. We set

$$
H=\left\{u \in L^{2}: \operatorname{div} u=0, \quad \int_{\mathbb{T}^{3}} u(x) \mathrm{d} x=0\right\} .
$$

Let us denote by $\Pi$ the orthogonal projection onto $H$ in $L^{2}$. Let $H^{k}$ be the space of vector functions $u=$ $\left(u_{1}, u_{2}, u_{3}\right)$ with components in the Sobolev space of order $k$, and let $\|\cdot\|_{k}$ be the corresponding norm. Define $H_{\sigma}^{k}:=H^{k} \cap H$. The Stokes operator is denoted by $L:=-\Pi \Delta, D(L)=H_{\sigma}^{2}$. For any vector $n=\left(n_{1}, n_{2}, n_{3}\right) \in \mathbb{R}^{3}$ we denote $|n|:=\left|n_{1}\right|+\left|n_{2}\right|+\left|n_{3}\right|$.

Let $J_{T}:=[0, T]$ and $X$ be a Banach space endowed with the norm $\|\cdot\|_{X}$. For $1 \leq p<\infty$ let $L^{p}\left(J_{T}, X\right)$ be the space of measurable functions $u: J_{T} \rightarrow X$ such that

$$
\|u\|_{L^{p}\left(J_{T}, X\right)}:=\left(\int_{0}^{T}\|u\|_{X}^{p} \mathrm{~d} s\right)^{\frac{1}{p}}<\infty .
$$

The space of continuous functions $u: J_{T} \rightarrow X$ is denoted by $C\left(J_{T}, X\right)$.

\section{Perturbative Result on solvability of the 3D Euler system}

Let us consider the Cauchy problem for Euler system on the 3D torus:

$$
\begin{aligned}
\dot{u}+\langle u, \nabla\rangle u+\nabla p & =f(t), \quad \operatorname{div} u=0, \\
u(0, x) & =u_{0}(x) .
\end{aligned}
$$


System (2.1), (2.2) is equivalent to the problem (see [15], Chap. 17)

$$
\begin{aligned}
\dot{v}+B(v) & =\Pi f(t), \\
v(0, x) & =\Pi u_{0}(x),
\end{aligned}
$$

where $v=\Pi u, B(a, b)=\Pi\{\langle a, \nabla\rangle b\}$ and $B(a)=B(a, a)$. We shall need the following standard estimates for the bilinear form $B$ :

$$
\begin{aligned}
\|B(a, b)\|_{k} \leq C\|a\|_{k}\|b\|_{k+1} & \text { for } k \geq 2, \\
\left|\left(B(a, b), L^{k} b\right)\right| \leq C\|a\|_{k}\|b\|_{k}^{2} & \text { for } k \geq 3,
\end{aligned}
$$

for any $a \in H_{\sigma}^{k}$ and $b \in H_{\sigma}^{k+1}$ (see [4]).

Let us consider the problem

$$
\begin{aligned}
\dot{u}+B(u+\zeta) & =f(t), \\
u(0, x) & =u_{0}(x)
\end{aligned}
$$

Theorem 2.1. Let $T>0$ and $k \geq 4$. Suppose that for some functions $v_{0} \in H_{\sigma}^{k}, \xi \in L^{2}\left(J_{T}, H_{\sigma}^{k+1}\right)$ and $g \in L^{1}\left(J_{T}, H_{\sigma}^{k}\right)$ problem (2.5), (2.6) with $u_{0}=v_{0}, \zeta=\xi$ and $f=g$ has a solution $v \in C\left(J_{T}\right.$, H $\left.H_{\sigma}^{k}\right)$. Then there are positive constants $\delta$ and $C$ depending only on the quantity

$$
\|v\|_{C\left(J_{T}, H^{k}\right)}+\|\xi\|_{L^{2}\left(J_{T}, H^{k}\right)}
$$

such that the following statements hold.

(i) If $u_{0} \in H_{\sigma}^{k}, \zeta \in L^{2}\left(J_{T}, H_{\sigma}^{k+1}\right)$ and $f \in L^{1}\left(J_{T}, H_{\sigma}^{k}\right)$ satisfy the inequalities

$$
\left\|v_{0}-u_{0}\right\|_{k-1}<\delta, \quad\|\zeta-\xi\|_{L^{2}\left(J_{T}, H^{k}\right)}<\delta, \quad\|f-g\|_{L^{1}\left(J_{T}, H^{k-1}\right)}<\delta
$$

then problem $(2.5),(2.6)$ has a unique solution $u \in C\left(J_{T}, H_{\sigma}^{k}\right)$.

(ii) Let

$$
\mathcal{R}: H_{\sigma}^{k} \times L^{2}\left(J_{T}, H_{\sigma}^{k+1}\right) \times L^{1}\left(J_{T}, H_{\sigma}^{k}\right) \rightarrow C\left(J_{T}, H_{\sigma}^{k}\right)
$$

be the operator that takes each triple $\left(u_{0}, \zeta, f\right)$ satisfying $(2.7)$ to the solution $u$ of (2.5), (2.6). Then

$\left\|\mathcal{R}\left(u_{0}, \zeta, f\right)-\mathcal{R}\left(v_{0}, \xi, g\right)\right\|_{C\left(J_{T}, H^{k-1}\right)} \leq C\left(\left\|v_{0}-u_{0}\right\|_{k-1}+\|\zeta-\xi\|_{L^{2}\left(J_{T}, H^{k}\right)}+\|f-g\|_{L^{1}\left(J_{T}, H^{k-1}\right)}\right)$

(iii) Let $\zeta \in C\left(J_{T}, H^{k}\right)$ and $f \in C\left(J_{T}, H^{k-1}\right)$, and let $\mathcal{R}_{t}$ be the restriction of $\mathcal{R}$ to the time $t$. Then $\mathcal{R}$. is Lipschitz-continuous in time, i.e.,

$$
\left\|\mathcal{R}_{t}\left(u_{0}, \zeta, f\right)-\mathcal{R}_{s}\left(u_{0}, \zeta, f\right)\right\|_{k-1} \leq M|t-s|
$$

where $M$ depends on $\left\|\mathcal{R}\left(u_{0}, \zeta, f\right)\right\|_{C\left(J_{T}, H^{k}\right)},\|\zeta\|_{C\left(J_{T}, H^{k}\right)}$ and $\|f\|_{C\left(J_{T}, H^{k-1}\right)}$.

Proof. We seek a solution of (2.5), (2.6) in the form $u=v+w$. Substituting this into (2.5), (2.6) and performing some transformations, we obtain the following problem for $w$ :

$$
\begin{aligned}
& \dot{w}+B(w+\eta, v+\xi)+B(v+\xi, w+\eta)+B(w+\eta)=q(t, x), \\
& w(0, x)=w_{0}(x)
\end{aligned}
$$


where $w_{0}=u_{0}-v_{0}, \eta=\zeta-\xi$ and $q=f-g$. By bilinearity of $B,(2.8)$ is equivalent to the equation

$$
\dot{w}+B(w)+\tilde{B}(w, \eta)+\tilde{B}(w, v)+\tilde{B}(w, \xi)=q(t, x)-(B(\eta)+\tilde{B}(v, \eta)+\tilde{B}(\xi, \eta)),
$$

where $\tilde{B}(u, v)=B(u, v)+B(v, u)$. It follows from (2.7) that we can choose $\delta>0$ such that the right-hand side of (2.10) and initial data $w_{0}$ are small in $L^{1}\left(J_{T}, H_{\sigma}^{k-1}\right)$ and $H_{\sigma}^{k-1}$, respectively. Hence, by the standard theorem of existence (see $[15,16])$, system $(2.10),(2.9)$ has a unique solution $w \in C\left(J_{T}, H_{\sigma}^{k-1}\right)$. From the embedding $H_{\sigma}^{2} \hookrightarrow L^{\infty}$ we deduce that

$$
\sup _{t \in[0, T]}\|\operatorname{rot} u(t, \cdot)\|_{L^{\infty}}<\infty .
$$

In view of $u_{0} \in H_{\sigma}^{k}, \zeta \in L^{2}\left(J_{T}, H_{\sigma}^{k+1}\right), f \in L^{1}\left(J_{T}, H_{\sigma}^{k}\right)$ and (2.11), the Beale-Kato-Majda theorem (see [3]) implies $u \in C\left(J_{T}, H_{\sigma}^{k}\right)$.

To prove (ii), let us get an a priori estimate for $w$. Multiplying (2.10) by $L^{k-1} w$ and using (2.3), (2.4), we obtain

$$
\begin{aligned}
\frac{1}{2} \frac{\mathrm{d}}{\mathrm{d} t}\|w\|_{k-1}^{2} \leq & C\left(\|w\|_{k-1}^{3}+\|w\|_{k-1}^{2}\left(\|\eta\|_{k}+\|v\|_{k}+\|\xi\|_{k}\right)\right. \\
& \left.+\|w\|_{k-1}\left(\|q\|_{k-1}+\|\eta\|_{k}\left(\|\eta\|_{k-1}+\|v\|_{k}+\|\xi\|_{k}\right)\right)\right) .
\end{aligned}
$$

Integrating (2.12), we obtain for any $t \in J_{T}$

$$
\|w(t)\|_{k-1}^{2} \leq C\|w\|_{C\left(J_{t}, H^{k-1}\right)}\left[\int_{0}^{t}\left(\|w\|_{k-1}^{2}+\|w\|_{k-1}\left(\|\eta\|_{k}+\|v\|_{k}+\|\xi\|_{k}\right)\right) \mathrm{d} s+A\right],
$$

where $A=\left\|w_{0}\right\|_{k-1}+\int_{0}^{T}\left[\|q\|_{k-1}+\|\eta\|_{k}\left(\|\eta\|_{k-1}+\|v\|_{k}+\|\xi\|_{k}\right)\right] \mathrm{d} s$. This implies that for any $\tau \in J_{t}$ we can estimate $\|w(\tau)\|_{k-1}^{2}$ by the right hand side of (2.13). Thus, we get

$$
\|w\|_{C\left(J_{t}, H^{k-1}\right)}^{2} \leq C\|w\|_{C\left(J_{t}, H^{k-1}\right)}\left[\int_{0}^{t}\left(\|w\|_{k-1}^{2}+\|w\|_{k-1}\left(\|\eta\|_{k}+\|v\|_{k}+\|\xi\|_{k}\right)\right) \mathrm{d} s+A\right] .
$$

Dividing (2.14) by $\|w\|_{C\left(J_{t}, H^{k-1}\right)}$, we get

$$
\begin{aligned}
\|w(t)\|_{k-1} & \leq\|w\|_{C\left(J_{t}, H^{k-1}\right)} \\
& \leq C\left[\int_{0}^{t}\left(\|w\|_{k-1}^{2}+\|w\|_{k-1}\left(\|\eta\|_{k}+\|v\|_{k}+\|\xi\|_{k}\right)\right) \mathrm{d} s+A\right] .
\end{aligned}
$$

The Gronwall inequality implies

$$
\|w(t)\|_{k-1} \leq A_{1}+C_{1} \int_{0}^{t}\|w(s, \cdot)\|_{k-1}^{2} \mathrm{~d} s
$$

where $A_{1}=C_{1} A$ and $C_{1}$ is a constant depending on $\|v\|_{C\left(J_{T}, H^{k}\right)}+\|\xi\|_{L^{2}\left(J_{T}, H^{k}\right)}$. Another application of Gronwall inequality gives that

$$
\|w(t)\|_{k-1} \leq \frac{A_{1}}{1-C_{1} A_{1} t} \leq 2 A_{1} \text { for any } t \leq \frac{1}{2 C_{1} A_{1}} .
$$


We can choose $\delta>0$ such that $\frac{1}{2 C_{1} A_{1}} \geq T$. From the definition of $A_{1}$ and (2.15) we deduce that

$$
\|w\|_{C\left(J_{T}, H^{k-1}\right)} \leq C\left(\left\|w_{0}\right\|_{H^{k-1}}+\|\eta\|_{L^{2}\left(J_{T}, H^{k}\right)}+\|q\|_{L^{1}\left(J_{T}, H^{k-1}\right)}\right) .
$$

Statement $(i i)$ is a straightforward consequence of (2.16).

Let us prove (iii). Integrating (2.5) over $(s, t)$ and using (2.3), we get

$$
\|u(t)-u(s)\|_{k-1} \leq \int_{s}^{t}\|f(\tau)-B(u(\tau)+\zeta(\tau))\|_{k-1} \mathrm{~d} \tau \leq M|t-s| .
$$

This completes the proof of Theorem 2.1.

\section{Controllability of the velocity}

Let us consider the controlled Euler system:

$$
\begin{aligned}
\dot{u}+B(u) & =h(t)+\eta(t), \\
u(0, x) & =u_{0}(x),
\end{aligned}
$$

where $h \in C^{\infty}\left([0, \infty), H_{\sigma}^{k+2}\right)$ and $u_{0} \in H_{\sigma}^{k}$ are given functions, and $\eta$ is the control taking values in a finitedimensional subspace $E \subset H_{\sigma}^{k+2}$. We denote by $\Theta\left(h, u_{0}\right)$ the set of functions $\eta \in L^{1}\left(J_{T}, H_{\sigma}^{k}\right)$ for which (3.1), (3.2) has a unique solution in $C\left(J_{T}, H_{\sigma}^{k}\right)$. By Theorem 2.1, $\Theta\left(h, u_{0}\right)$ is an open subset of $L^{1}\left(J_{T}, H_{\sigma}^{k}\right)$. To simplify the notation, we write $\mathcal{R}(\cdot, 0, \cdot)=\mathcal{R}(\cdot, \cdot)$. Let us recall the definition of controllability. Suppose $X \subset L^{1}\left(J_{T}, H_{\sigma}^{k}\right)$ is an arbitrary vector space.

Definition 3.1. Equation (3.1) with $\eta \in X$ is said to be controllable at time $T$ if for any $\varepsilon>0$, for any finite-dimensional subspace $F \subset H_{\sigma}^{k}$, for any projection $P_{F}: H_{\sigma}^{k} \rightarrow H_{\sigma}^{k}$ onto $F$ and for any functions $u_{0} \in H_{\sigma}^{k}$, $\hat{u} \in H_{\sigma}^{k}$ there is a control $\eta \in \Theta\left(h, u_{0}\right) \cap X$ such that

$$
\begin{aligned}
P_{F} \mathcal{R}_{T}\left(u_{0}, \eta\right) & =P_{F} \hat{u} \\
\left\|\mathcal{R}_{T}\left(u_{0}, \eta\right)-\hat{u}\right\|_{k} & <\varepsilon .
\end{aligned}
$$

Let us recall some notation introduced in [1,2,12]. For any finite-dimensional subspace $E \subset H_{\sigma}^{k+2}$, we denote by $\mathcal{F}(E)$ the largest vector space $\mathcal{F} \subset H_{\sigma}^{k+2}$ such that for any $\eta_{1} \in F$ there are vectors $\eta, \zeta^{1}, \ldots, \zeta^{n} \in E$ and positive constants $\alpha_{1}, \ldots, \alpha_{n}$ satisfying the relation

$$
\eta_{1}=\eta-\sum_{i=1}^{n} \alpha_{i} B\left(\zeta^{i}\right)
$$

The space $\mathcal{F}(E)$ is well defined. Indeed, as $E$ is a finite-dimensional subspace and $B$ is a bilinear operator, then $\mathcal{F}(E)$ is contained in a finite-dimensional space. It is easy to see that if subspaces $G_{1}$ and $G_{2}$ satisfy (3.3), then so does $G_{1}+G_{2}$. Thus, $\mathcal{F}(E)$ is well defined. Obviously, $E \subset \mathcal{F}(E)$. We define $E_{k}$ by the rule

$$
E_{0}=E, \quad E_{n}=\mathcal{F}\left(E_{n-1}\right) \quad \text { for } \quad n \geq 1, \quad E_{\infty}=\bigcup_{n=1}^{\infty} E_{n} .
$$

The following theorem is the main result of this section.

Theorem 3.2. Let $h \in C^{\infty}\left([0, \infty), H_{\sigma}^{k+2}\right)$. If $E \subset H_{\sigma}^{k+2}$ is a finite-dimensional subspace such that $E_{\infty}$ is dense in $H_{\sigma}^{k}$, then equation (3.1) with $\eta \in C^{\infty}\left(J_{T}, E\right)$ is controllable at any time $T$. 
Example 3.3. Let us introduce the functions

$$
c_{m}(x)=l(m) \cos \langle m, x\rangle, s_{m}(x)=l(m) \sin \langle m, x\rangle,
$$

where $m \in \mathbb{Z}^{3}$ and

$$
\{l(m), l(-m)\} \text { is an orthonormal basis in } m^{\perp}:=\left\{x \in \mathbb{R}^{3},\langle x, m\rangle=0\right\} .
$$

It is shown in [12] that if

$$
E=\operatorname{span}\left\{c_{m}, s_{m},|m| \leq 3\right\},
$$

then $E_{\infty}$ is dense in $H_{\sigma}^{k}$. We emphasize for what follows that the space $E$ does not depend on the choice of the basis $\{l(m), l(-m)\}$.

The proof of Theorem 3.2 is based on the uniform approximate controllability of the Euler system.

Definition 3.4. Equation (3.1) with $\eta \in X$ is said to be uniformly approximately controllable at time $T$ if for any $\varepsilon>0$, any $u_{0} \in H_{\sigma}^{k}$ and any compact set $K \subset H_{\sigma}^{k}$ there is a continuous function $\Psi: K \rightarrow \Theta\left(h, u_{0}\right) \cap X$ such that

$$
\sup _{\hat{u} \in K}\left\|\mathcal{R}_{T}\left(u_{0}, \Psi(\hat{u})\right)-\hat{u}\right\|_{k}<\varepsilon
$$

where $\Theta\left(h, u_{0}\right) \cap X$ is endowed with the norm of $L^{1}\left(J_{T}, H^{k}\right)$.

The following lemma shows that to prove uniform approximate controllability of equation (3.1) it suffices to consider the case in which the target set $K \subset H_{\sigma}^{k}$ consists of sufficiently smooth functions.

Lemma 3.5. Suppose that for compact subset $K \subset H_{\sigma}^{k+1}$ there is a continuous function $\Psi: K \rightarrow \Theta\left(h, u_{0}\right) \cap X$ such that (3.6) holds. Then equation (3.1) with $\eta \in X$ is uniformly approximately controllable at time $T$.

Proof. For any compact set $K \subset H_{\sigma}^{k}$ there is a small constant $\delta>0$ such that

$$
\sup _{\hat{u} \in K}\left\|\mathrm{e}^{-\delta L} \hat{u}-\hat{u}\right\|_{k}<\frac{\varepsilon}{2} \text {. }
$$

As $K_{1}:=\mathrm{e}^{-\delta L} K$ is compact in $H_{\sigma}^{k+1}$, by assumption, there is a continuous mapping $\Psi: K_{1} \rightarrow \Theta\left(h, u_{0}\right) \cap$ $C^{\infty}\left(J_{T}, X\right)$ such that

$$
\sup _{\hat{u} \in K_{1}}\left\|\mathcal{R}_{T}\left(u_{0}, \Psi(\hat{u})\right)-\hat{u}\right\|_{k}<\frac{\varepsilon}{2} .
$$

Therefore the continuous mapping $\Phi: K \rightarrow \Theta\left(h, u_{0}\right) \cap X, \hat{u} \rightarrow \Psi\left(\mathrm{e}^{-\delta L} \hat{u}\right)$ satisfies the inequality

$$
\sup _{\hat{u} \in K}\left\|\mathcal{R}_{T}\left(u_{0}, \Phi(\hat{u})\right)-\hat{u}\right\|_{k}<\varepsilon .
$$

The following lemma shows that the uniform approximate controllability is stronger than controllability.

Lemma 3.6. If equation (3.1) with $\eta \in X$ is uniformly approximately controllable at time $T$, then it is also controllable.

Proof. Suppose $F \subset H_{\sigma}^{k}$ is a finite-dimensional subspace and $P_{F}$ is a projection onto $F, u_{0} \in H_{\sigma}^{k}$ and $\hat{u} \in F$. Let $B_{F}(R)$ be the closed ball in $F$ of radius $R$ centred at origin with $R>M \varepsilon$, where $M$ is the norm of $P_{F}$ and $\varepsilon>0$ is an arbitrary constant. Since $B_{F}(R)$ is a compact subset of $H_{\sigma}^{k}$, there is a continuous mapping $\Psi: B_{F}(R) \rightarrow \Theta\left(h, u_{0}\right) \cap X$ such that

$$
\sup _{\hat{u} \in B_{F}(R)}\left\|\mathcal{R}_{T}\left(u_{0}, \Psi(\hat{u})\right)-\hat{u}\right\|_{k}<\varepsilon .
$$


Therefore the continuous mapping $\Phi: B_{F}(R) \rightarrow F, \hat{u} \rightarrow P_{F} \mathcal{R}_{T}\left(u_{0}, \Psi(\hat{u})\right)$ satisfies the inequality

$$
\sup _{\hat{u} \in B_{F}(R)}\|\Phi(\hat{u})-\hat{u}\|_{k}<M \varepsilon .
$$

Fixing $v \in B_{F}(R-M \varepsilon)$ and applying the Brouwer theorem to the mapping $u \rightarrow v+u-\Phi(u): B_{F}(R) \rightarrow B_{F}(R)$, we get

$$
B_{F}(R-M \varepsilon) \subset \Phi\left(B_{F}(R)\right) .
$$

Let $\hat{u} \in F$. By (3.8), for sufficiently large $R$ there is a function $u_{1} \in B_{F}(R)$ such that

$$
P_{F} \mathcal{R}_{T}\left(u_{0}, \Psi\left(u_{1}\right)\right)=\hat{u} .
$$

Using (3.7) and (3.9), we obtain

$$
\left\|\mathcal{R}_{T}\left(u_{0}, \Psi\left(u_{1}\right)\right)-\hat{u}\right\|_{k} \leq\left\|\mathcal{R}_{T}\left(u_{0}, \Psi\left(u_{1}\right)\right)-u_{1}\right\|_{k}+\left\|u_{1}-P_{F} \mathcal{R}_{T}\left(u_{0}, \Psi\left(u_{1}\right)\right)\right\|_{k}<\varepsilon+M \varepsilon .
$$

Since $\varepsilon>0$ was arbitrary, this completes the proof.

Lemma 3.6 implies that Theorem 3.2 is an immediate consequence of the following result, which will be proved in Sections 5 and 6.

Theorem 3.7. Let $h \in C^{\infty}\left([0, \infty), H_{\sigma}^{k+2}\right)$. If $E \subset H_{\sigma}^{k+2}$ is a finite-dimensional subspace such that $E_{\infty}$ is dense in $H_{\sigma}^{k}$, then equation (3.1) with $\eta \in C^{\infty}\left(J_{T}, E\right)$ is uniformly approximately controllable at any time $T$.

\section{Controllability of Finite-Dimensional projections of the Velocity AND PRESSURE}

In this section, we are interested in controllability properties of pressure in Euler system. We consider the problem (1.1), (1.2). If $u \in C\left(J_{T}, H^{k}\right)$ is a solution of (3.1), (3.2), then $(u, p)$ will be the solution of (1.1), (1.2), where

$$
p=\Delta^{-1}\left(\operatorname{div} h-\sum_{i, j=1}^{3} \partial_{j} u_{i} \partial_{i} u_{j}\right) .
$$

Here the function $p$ is defined up to the an additive constant and $\Delta^{-1}$ is the inverse of $\Delta: H_{\sigma}^{k} \rightarrow: H_{\sigma}^{k-2}$. In what follows we normalize $p$ by the condition that its mean value on $\mathbb{T}^{3}$ is zero. Denote by $\left(\mathcal{R}\left(u_{0}, \eta\right), \mathcal{P}\left(u_{0}, \eta\right)\right)$ the solution of $(1.1),(1.2)$ and by $\left(\mathcal{R}_{t}\left(u_{0}, \eta\right), \mathcal{P}_{t}\left(u_{0}, \eta\right)\right)$ its restriction to the time $t$. Equation (4.1) implies that (1.1), (1.2) is not approximately controllable, so we will be interested in exact controllability in projections.

Definition 4.1. Equation (1.1) with $\eta \in X$ is said to be exactly controllable in projections at time $T$ if for any finite-dimensional subspaces $F \subset H_{\sigma}^{k}, G \subset H^{k}$ and for any functions $u_{0} \in H_{\sigma}^{k}, \hat{u} \in F$ and $\hat{p} \in G$ there is a control $\eta \in \Theta\left(h, u_{0}\right) \cap X$ such that

$$
\begin{aligned}
& P_{F} \mathcal{R}_{T}\left(u_{0}, \eta\right)=\hat{u}, \\
& P_{G} \mathcal{P}_{T}\left(u_{0}, \eta\right)=\hat{p} .
\end{aligned}
$$

Theorem 4.2. If $E \subset H_{\sigma}^{k+2}$ is a finite-dimensional subspace such that $E_{\infty}$ is dense in $H_{\sigma}^{k}$, then equation (1.1) with $\eta \in C^{\infty}\left(J_{T}, E\right)$ is exactly controllable in projections at any time $T>0$. 
Proof. To simplify the proof, we shall assume that $h=0$. The proof remains literally the same in the case $h \neq 0$. An argument similar to that used in the proof of Lemma 3.6 shows that it suffices to establish the following property: for any compact set $K \subset H_{\sigma}^{k} \times H^{k}$ and for any constant $\varepsilon>0$ there is a continuous function $\Psi: K \rightarrow \Theta\left(h, u_{0}\right) \cap X$ such that

$$
\begin{array}{r}
\sup _{(\hat{u}, \hat{p}) \in K}\left\|\mathcal{R}_{T}\left(u_{0}, \Psi(\hat{u}, \hat{p})\right)-\hat{u}\right\|_{k}<\varepsilon, \\
\sup _{(\hat{u}, \hat{p}) \in K}\left\|P_{G} \mathcal{P}_{T}\left(u_{0}, \Psi(\hat{u}, \hat{p})\right)-\hat{p}\right\|_{k}<\varepsilon .
\end{array}
$$

We introduce the spaces

$$
\begin{aligned}
F_{m}: & =\operatorname{span}\left\{c_{n}, s_{n},|n| \leq m, n \in \mathbb{Z}_{*}^{3}\right\}, \\
G_{m}: & =\operatorname{span}\left\{\sin \langle n, x\rangle, \cos \langle n, x\rangle,|n| \leq m, n \in \mathbb{Z}_{*}^{3}\right\},
\end{aligned}
$$

where the functions $c_{n}, s_{n}$ are defined in (3.4), (3.5). By an approximation argument, it suffices to construct $\Psi$ for any compact set $K \subset F_{m} \times G_{m}$. For an integer $m \geq 1$, we introduce the symmetric quadratic form

$$
A(u, v)=-P_{G_{m}} \Delta^{-1} \sum_{i, j=1}^{3} \partial_{j} u_{i} \partial_{i} v_{j}
$$

and set $A(u)=A(u, u)$. Clearly, we have the following inequality

$$
\|A(u)-A(v)\|_{k} \leq C\|u-v\|_{k},
$$

where $u, v \in H_{\sigma}^{k}$ and $C$ is constant depending on $\|u\|_{k}+\|v\|_{k}$. Equation (4.1) implies

$$
P_{G_{m}} \mathcal{P}_{t}\left(u_{0}, \eta\right)=A\left(\mathcal{R}_{t}\left(u_{0}, \eta\right)\right) .
$$

We admit for the moment the following lemma.

Lemma 4.3. For any $\hat{u} \in F_{m}$ and $\hat{p} \in G_{m}$ there is $v \in F_{m}^{\perp} \cap H_{\sigma}^{k}$ such that

$$
\hat{p}=A(\hat{u}+v),
$$

where $F_{m}^{\perp}$ is the orthogonal complement of $F_{m}$ in the space $H$. Moreover, the mapping $(\hat{u}, \hat{p}) \rightarrow v$ is continuous from $F_{m} \times G_{m}$ to $F_{m}^{\perp}$, where $F_{m}, G_{m}$ and $F_{m}^{\perp}$ are endowed with the norm of $H^{k}$.

By Theorem 3.7, there is a continuous mapping $\Psi$ such that

$$
\sup _{(\hat{u}, \hat{p}) \in K}\left\|\mathcal{R}_{T}\left(u_{0}, \Psi(\hat{u}, \hat{p})\right)-(\hat{u}+v)\right\|_{k}<\varepsilon,
$$

where $v$ satisfies (4.4). From (4.2), (4.3) and (4.4), we have

$$
\begin{aligned}
\sup _{(\hat{u}, \hat{p}) \in K}\left\|P_{G_{m}} \mathcal{P}_{T}\left(u_{0}, \Psi(\hat{u}, \hat{p})\right)-\hat{p}\right\|_{k} & \leq \sup _{(\hat{u}, \hat{p}) \in K}\left\|A\left(\mathcal{R}_{T}\left(u_{0}, \Psi(\hat{u}, \hat{p})\right)\right)-A(\hat{u}+v)\right\|_{k} \\
& \leq C \sup _{(\hat{u}, \hat{p}) \in K}\left\|\mathcal{R}_{T}\left(u_{0}, \Psi(\hat{u}, \hat{p})\right)-(\hat{u}+v)\right\|_{k} .
\end{aligned}
$$

This completes the proof of Theorem 4.2. 
Proof of Lemma 4.3. It is easy to see that (4.4) is equivalent to

$$
A(v)+2 A(\hat{u}, v)=\hat{p}-A(\hat{u})=: \sum_{|n| \leq m}\left(C_{n} \sin \langle n, x\rangle+D_{n} \cos \langle n, x\rangle\right) .
$$

For all $n \in \mathbb{Z}_{*}^{3},|n| \leq m$ let us take $\left\{k_{n}^{1}\right\},\left\{k_{n}^{2}\right\},\left\{k_{n}^{3}\right\}$ and $\left\{k_{n}^{4}\right\}$ in $\mathbb{Z}_{*}^{3}$ such that $\left|k_{n}^{i}\right|>2 m$ and

(a) $k_{n}^{2}-k_{n}^{1}=k_{n}^{4}-k_{n}^{3}=n$;

(b) $\min \left\{\left|k_{n}^{i}+k_{n}^{j}\right|,\left|k_{n}^{i} \pm k_{r}^{j}\right|,\left|k_{n}^{3}-k_{n}^{d}\right|,\left|k_{n}^{4}-k_{n}^{d}\right|\right\}>m$;

(c) $k_{n}^{1}$ and $k_{n}^{3}$ are not parallel to $k_{n}^{2}$ and $k_{n}^{4}$, respectively,

for all $i, j=1,2,3,4, d=1,2,|r|<m$ and $n \neq r$. This choice is possible. Indeed, let $\phi: \mathbb{Z}_{*}^{3} \rightarrow \mathbb{N}_{*}$ be an injection and let

$$
\begin{array}{ll}
k_{n}^{1}=8 \phi(n) \mathfrak{m}(n), & k_{n}^{3}=(8 \phi(n)+4) \mathfrak{m}(n), \\
k_{n}^{2}=8 \phi(n) \mathfrak{m}(n)+n, & k_{n}^{4}=(8 \phi(n)+4) \mathfrak{m}(n)+n,
\end{array}
$$

where $\mathfrak{m}(n) \in \mathbb{Z}_{*}^{3}$ is not parallel to $n$ and $|\mathfrak{m}(n)|=m$. It is easy to see that $\left\{k_{n}^{j}\right\}$ satisfy $(a)-(c)$. We seek $v$ in the form

$$
v=\sum_{|n| \leq m}\left(C_{k_{n}^{1}} s_{k_{n}^{1}}+D_{k_{n}^{2}} c_{k_{n}^{2}}+C_{k_{n}^{3}} s_{k_{n}^{3}}+C_{k_{n}^{4}} s_{k_{n}^{4}}\right) .
$$

Substituting this expression of $v$ into (4.5) and using the construction of $k_{n}^{i}$, we obtain

$$
\sum_{|n| \leq m}\left(A\left(C_{k_{n}^{1}} s_{k_{n}^{1}}+D_{k_{n}^{2}} c_{k_{n}^{2}}\right)+A\left(C_{k_{n}^{3}} s_{k_{n}^{3}}+C_{k_{n}^{4}} s_{k_{n}^{4}}\right)\right)=\sum_{|n| \leq m}\left(C_{n} \sin \langle n, x\rangle+D_{n} \cos \langle n, x\rangle\right) .
$$

On the other hand,

$$
\begin{aligned}
A\left(C_{k_{n}^{1}} s_{k_{n}^{1}}+D_{k_{n}^{2}} c_{k_{n}^{2}}\right) & =\Delta^{-1} \sum_{i, j=1}^{3} l_{i}\left(k_{n}^{1}\right)\left(k_{n}^{1}\right)_{j} l_{j}\left(k_{n}^{2}\right)\left(k_{n}^{2}\right)_{i} C_{k_{n}^{1}} D_{k_{n}^{2}} \sin \langle n, x\rangle \\
& =-\frac{C_{k_{n}^{1}} D_{k_{n}^{2}}}{n_{1}^{2}+n_{2}^{2}+n_{3}^{2}}\left\langle l\left(k_{n}^{1}\right), k_{n}^{2}\right\rangle\left\langle l\left(k_{n}^{2}\right), k_{n}^{1}\right\rangle \sin \langle n, x\rangle,
\end{aligned}
$$

where $l_{j}\left(k_{n}^{i}\right)$ and $\left(k_{n}^{i}\right)_{j}$ are $j$ th coordinates of $l\left(k_{n}^{i}\right)$ and $k_{n}^{i}$, respectively. As $k_{n}^{1}$ is not parallel to $k_{n}^{2}$, we can choose $l\left(k_{n}^{1}\right)$ and $l\left(k_{n}^{2}\right)$ not perpendicular to $k_{n}^{2}$ and $k_{n}^{1}$, respectively, i.e.,

$$
\left\langle l\left(k_{n}^{1}\right), k_{n}^{2}\right\rangle\left\langle l\left(k_{n}^{2}\right), k_{n}^{1}\right\rangle \neq 0 .
$$

Hence, there are constants $C_{k_{n}^{1}}, D_{k_{n}^{2}}$ continuously depending on $C_{n}$, and therefore on $(\hat{u}, \hat{p})$, such that

$$
A\left(C_{k_{n}^{1}} s_{k_{n}^{1}}+D_{k_{n}^{2}} c_{k_{n}^{2}}\right)=C_{n} \sin \langle n, x\rangle .
$$

In the same way, we can choose $C_{k_{n}^{3}}, C_{k_{n}^{4}}$ such that

$$
A\left(C_{k_{n}^{3}} s_{k_{n}^{3}}+C_{k_{n}^{4}} s_{k_{n}^{4}}\right)=D_{n} \cos \langle n, x\rangle .
$$

Thus we have (4.4). 


\section{Proof of Theorem 3.7}

Let us fix a constant $\varepsilon>0$, an initial point $u_{0} \in H_{\sigma}^{k}$, a compact set $K \subset H_{\sigma}^{k}$ and a vector subspace $X \subset L^{1}\left(J_{T}, H_{\sigma}^{k}\right)$. Equation (3.1) with $\eta \in X$ is said to be uniformly $\left(\varepsilon, u_{0}, K\right)$-controllable at time $T>0$ if there is a continuous mapping

$$
\Psi: K \rightarrow \Theta\left(h, u_{0}\right) \cap X
$$

such that

$$
\sup _{\hat{u} \in K}\left\|\mathcal{R}_{T}\left(u_{0}, \Psi(\hat{u})\right)-\hat{u}\right\|_{k}<\varepsilon
$$

where $\Theta\left(h, u_{0}\right) \cap X$ is endowed with the norm of $L^{1}\left(J_{T}, H_{\sigma}^{k}\right)$.

Theorem 3.7 is deduced from the following result, which is established in next section.

Theorem 5.1. Let $E \subset H_{\sigma}^{k+2}$ be a finite-dimensional subspace. If equation (3.1) with $\eta \in C^{\infty}\left(J_{T}, \mathcal{F}(E)\right)$ is uniformly $\left(\varepsilon, u_{0}, K\right)$-controllable, then it is also $\left(\varepsilon, u_{0}, K\right)$-controllable with $\eta \in C^{\infty}\left(J_{T}, E\right)$.

Proof of Theorem 3.7. We first prove that there is an integer $N \geq 1$ depending only on $\varepsilon, u_{0}$ and $K$ such that equation (3.1) with $\eta \in C\left(J_{T}, E_{N}\right)$ is uniformly $\left(\varepsilon, u_{0}, K\right)$-controllable at time $T$. Let us define a continuous operator defined on $J_{T} \times K$ by

$$
u_{\mu, \delta}(t, \hat{u})=T^{-1}\left(t \mathrm{e}^{\mu L} \hat{u}+(T-t) \mathrm{e}^{\delta L} u_{0}\right) .
$$

It is easy to see that $u_{\mu, \delta}$ satisfies equation (3.1) with

$$
\eta_{\mu, \delta}=\dot{u}_{\mu, \delta}+B\left(u_{\mu, \delta}\right)-h(t)
$$

As $K$ is a compact set in $H_{\sigma}^{k}$, we have

$$
\begin{aligned}
& \sup _{\hat{u} \in K}\left\|u_{\mu, \delta}(T, \hat{u})-\hat{u}\right\|_{k} \rightarrow 0 \text { as } \mu \rightarrow 0, \\
& \sup _{\hat{u} \in K}\left\|u_{\mu, \delta}(0, \hat{u})-u_{0}\right\|_{k} \rightarrow 0 \text { as } \delta \rightarrow 0 .
\end{aligned}
$$

The fact that $E_{\infty}$ is dense in $H_{\sigma}^{k}$ implies

$$
\left\|P_{E_{N}} \eta_{\mu, \delta}-\eta_{\mu, \delta}\right\|_{L^{1}\left(J_{T}, H^{k}\right)} \rightarrow 0 \text { as } N \rightarrow \infty .
$$

By Theorem 2.1, we can chose $N, \mu$ and $\delta$ such that

$$
\sup _{\hat{u} \in K}\left\|\mathcal{R}\left(u_{0}, P_{E_{N}} \eta_{\mu, \delta}(\hat{u})\right)-\hat{u}\right\|_{k}<\varepsilon .
$$

We note that the mapping $P_{E_{N}} \eta_{\mu, \delta}(\cdot, \cdot): \hat{u} \rightarrow P_{E_{N}} \eta_{\mu, \delta}(\cdot, \hat{u})$ is continuous from $K$ to $C\left(J_{T}, H_{\sigma}^{k}\right)$. Hence equation (3.1) is uniformly $\left(\varepsilon, u_{0}, K\right)$-controllable with $\eta \in C\left(J_{T}, E_{N}\right)$. Applying $N$ times Theorem 5.1, we complete the proof of Theorem 3.7 .

\section{Proof of Theorem 5.1}

The proof of Theorem 5.1 is inspired by ideas from $[1,2,12,13]$. Let us consider the following control system:

$$
\dot{u}+B(u+\zeta)=h+\eta
$$


where $\eta, \zeta$ are $E$-valued controls. Let $\hat{\Theta}\left(u_{0}, h\right)$ be the set of pairs $(\eta, \zeta) \in L^{1}\left(J_{T}, H_{\sigma}^{k}\right) \times L^{2}\left(J_{T}, H_{\sigma}^{k+1}\right)$ for which problem (6.1), (3.2) has a unique solution in $C\left(J_{T}, H_{\sigma}^{k}\right)$. Equation (6.1) with $(\eta, \zeta) \in \hat{X} \subset L^{1}\left(J_{T}, H_{\sigma}^{k}\right) \times$ $L^{2}\left(J_{T}, H_{\sigma}^{k+1}\right)$ is said to be uniformly $\left(\varepsilon, u_{0}, K\right)$-controllable if there is a continuous mapping

$$
\hat{\Psi}: K \rightarrow \hat{\Theta}\left(h, u_{0}\right) \cap \hat{X}
$$

such that

$$
\sup _{\hat{u} \in K}\left\|\mathcal{R}_{T}\left(u_{0}, \hat{\Psi}(\hat{u})\right)-\hat{u}\right\|_{k}<\varepsilon
$$

where $\hat{\Theta}\left(h, u_{0}\right) \cap \hat{X}$ is endowed with the norm of $L^{1}\left(J_{T}, H_{\sigma}^{k}\right) \times L^{2}\left(J_{T}, H_{\sigma}^{k+1}\right)$.

We claim that, when proving Theorem 5.1, it suffices to assume $u_{0} \in H_{\sigma}^{k+2}$. Suppose that for any $v_{0} \in H_{\sigma}^{k+2}$ and for any continuous mapping $\Phi: K \rightarrow \Theta\left(h, v_{0}\right) \cap C^{\infty}\left(J_{T}, E_{1}\right)$ there is a continuous mapping

$$
\hat{\Phi}: K \rightarrow \Theta\left(h, v_{0}\right) \cap C^{\infty}\left(J_{T}, E\right)
$$

such that

$$
\sup _{\hat{u} \in K}\left\|\mathcal{R}_{T}\left(v_{0}, \Phi(\hat{u})\right)-\mathcal{R}_{T}\left(v_{0}, \hat{\Phi}(\hat{u})\right)\right\|_{k}<\frac{\varepsilon}{3}
$$

Let us show that for any $u_{0} \in H_{\sigma}^{k}$ and for any continuous mapping $\Psi: K \rightarrow \Theta\left(h, v_{0}\right) \cap C^{\infty}\left(J_{T}, E_{1}\right)$ there is a continuous mapping $\hat{\Phi}: K \rightarrow \Theta\left(h, v_{0}\right) \cap C^{\infty}\left(J_{T}, E\right)$ such that

$$
\sup _{\hat{u} \in K}\left\|\mathcal{R}_{T}\left(u_{0}, \Psi(\hat{u})\right)-\mathcal{R}_{T}\left(u_{0}, \hat{\Psi}(\hat{u})\right)\right\|_{k}<\varepsilon .
$$

By Theorem 2.1, there is $v_{0} \in H_{\sigma}^{k+2}$ such that

$$
\sup _{\hat{u} \in K}\left\|\mathcal{R}\left(u_{0}, \Psi(\hat{u})\right)-\mathcal{R}\left(v_{0}, \Psi(\hat{u})\right)\right\|_{C\left(J_{T}, H^{k}\right)}<\frac{\varepsilon}{3} .
$$

By our assumption, as $v_{0} \in H_{\sigma}^{k+2}$, there is a continuous mapping

$$
\hat{\Psi}_{\varepsilon}: K \rightarrow \Theta\left(h, u_{0}\right) \cap C^{\infty}\left(J_{T}, E\right)
$$

such that

$$
\sup _{\hat{u} \in K}\left\|\mathcal{R}_{T}\left(v_{0}, \Psi(\hat{u})\right)-\mathcal{R}_{T}\left(v_{0}, \hat{\Psi}_{\varepsilon}(\hat{u})\right)\right\|_{k}<\frac{\varepsilon}{3} .
$$

By Theorem 2.1, we have

$$
\left\|\mathcal{R}_{T}\left(v_{0}, \hat{\Psi}_{\varepsilon}(\hat{u})\right)-\mathcal{R}_{T}\left(u_{0}, \hat{\Psi}_{\varepsilon}(\hat{u})\right)\right\|_{k} \leq C\left\|v_{0}-u_{0}\right\|
$$

where $C$ is a constant not depending on $\varepsilon$. Choosing $v_{0}$ sufficiently close to $u_{0}$ and using inequalities (6.3), (6.4) and (6.5), we get

$$
\left\|\mathcal{R}_{T}\left(u_{0}, \Psi(\hat{u})\right)-\mathcal{R}_{T}\left(u_{0}, \hat{\Psi}_{\varepsilon}(\hat{u})\right)\right\|_{k}<\varepsilon .
$$

From now on, we assume that $u_{0} \in H_{\sigma}^{k+2}$. In this case, Theorem 5.1 is deduced from the following two propositions.

Proposition 6.1. Equation (3.1) with $\eta \in C^{\infty}\left(J_{T}, E\right)$ is uniformly $\left(\varepsilon, u_{0}, K\right)$-controllable if and only if so is equation (6.1) with $(\eta, \zeta) \in C^{\infty}\left(J_{T}, E \times E\right)$. 
Proposition 6.2. Equation (6.1) with $(\eta, \zeta) \in C^{\infty}\left(J_{T}, E \times E\right)$ is uniformly $\left(\varepsilon, u_{0}, K\right)$-controllable if and only if so is equation (3.1) with $\eta_{1} \in C^{\infty}\left(J_{T}, E_{1}\right)$.

Proof of Proposition 6.1. We show that if (6.1) with $(\eta, \zeta) \in C^{\infty}\left(J_{T}, E \times E\right)$ is uniformly $\left(\varepsilon, u_{0}, K\right)$-controllable, then so is (3.1) with $\eta \in C^{\infty}\left(J_{T}, E\right)$. Let

$$
\hat{\Psi}: K \rightarrow \hat{\Theta}\left(h, u_{0}\right) \cap C^{\infty}\left(J_{T}, E \times E\right), \quad \hat{\Psi}(\hat{u})=(\eta(t, \hat{u}), \zeta(t, \hat{u}))
$$

be such that

$$
\hat{\varepsilon}:=\sup _{\hat{u} \in K}\left\|\mathcal{R}_{T}\left(u_{0}, \hat{\Psi}(\hat{u})\right)-\hat{u}\right\|_{k}<\varepsilon .
$$

Let us choose $\zeta_{n}(\cdot, \hat{u}) \in C^{\infty}\left(J_{T}, E\right)$ such that $\zeta_{n}(0)=\zeta_{n}(T)=0$, the mapping $\zeta_{n}(\cdot, \cdot): \hat{u} \rightarrow \zeta_{n}(\cdot, \hat{u})$ from $K$ to $C^{1}\left(J_{T}, H_{\sigma}^{k+1}\right)$ is continuous and

$$
\left\|\zeta_{n}-\zeta\right\|_{L^{2}\left(J_{T}, H^{k+1}\right)} \rightarrow 0 \text { as } n \rightarrow \infty .
$$

By Theorem 2.1, for sufficiently large $n$ we have

$$
\sup _{\hat{u} \in K}\left\|\mathcal{R}_{T}\left(u_{0}, \zeta_{n}(\hat{u}), \eta\right)-\mathcal{R}_{T}\left(u_{0}, \hat{\Psi}(\hat{u})\right)\right\|_{k}<\varepsilon-\hat{\varepsilon} .
$$

Define $\Psi_{n}(t, \hat{u})=\eta(t, \hat{u})-\dot{\zeta}_{n}(t, \hat{u})$. It is easy to see that $\Psi_{n}(\cdot, \cdot): \hat{u} \rightarrow \Psi_{n}(\cdot, \hat{u})$ is a continuous mapping from $K$ to $L^{1}\left(J_{T}, H_{\sigma}^{k}\right)$. Clearly,

$$
\mathcal{R}\left(u_{0}, \zeta_{n}(\hat{u}), \eta\right)=\mathcal{R}\left(u_{0}, \Psi_{n}(\hat{u})\right)-\zeta_{n}(\hat{u}) .
$$

Using the fact that $\zeta_{n}(T)=0,(6.7)$ and (6.6), we derive

$$
\sup _{\hat{u} \in K}\left\|\mathcal{R}_{T}\left(u_{0}, \Psi_{n}(\hat{u})\right)-\hat{u}\right\|_{k}<\varepsilon-\hat{\varepsilon}+\sup _{\hat{u} \in K}\left\|\mathcal{R}_{T}\left(u_{0}, \hat{\Psi}(\hat{u})\right)-\hat{u}\right\|_{k}<\varepsilon,
$$

which completes the proof of Proposition 6.1.

Proof of Proposition 6.2. By Proposition 6.1 and the fact $E \subset E_{1}$, if equation (6.1) is uniformly $\left(\varepsilon, u_{0}, K\right)$ controllable, then so is equation (3.1) with $\eta \in C^{\infty}\left(J_{T}, E_{1}\right)$. We need to prove the converse assertion. We assume that there is a continuous mapping

$$
\Psi_{1}: K \rightarrow \Theta\left(h, u_{0}\right) \cap L^{1}\left(J_{T}, E_{1}\right)
$$

such that

$$
\hat{\varepsilon}:=\sup _{\hat{u} \in K}\left\|\mathcal{R}_{T}\left(u_{0}, \Psi_{1}(\hat{u})\right)-\hat{u}\right\|_{k}<\varepsilon .
$$

We approximate $\mathcal{R}_{T}\left(u_{0}, \Psi_{1}(\hat{u})\right)$ by a solution $u(t, \hat{u})$ of problem $(6.1),(3.2)$ with some $\eta(t, \hat{u}), \zeta(t, \hat{u}) \in$ $C^{\infty}\left(J_{T}, E\right)$ such that $(\eta(t, \hat{u}), \zeta(t, \hat{u}))$ depends continuously on $\hat{u} \in K$.

Step 1. We first approximate $\Psi_{1}(\hat{u})$ by a family of piecewise constant controls. Let us introduce a finite set $A=\left\{\eta_{1}^{l} \in E_{1}, l=1, \ldots, m\right\}$. For any integer $s$, we denote by $P_{s}\left(J_{T}, A\right)$ the set of functions

$$
\eta_{1}(t)=\sum_{l=1}^{m} \varphi_{l}(t) \eta_{1}^{l} \text { for } t \in[0, T]
$$


where $\varphi_{l}$ are non-negative functions such that $\sum_{l=1}^{m} \varphi_{l}(t)=1$,

$$
\varphi_{l}(t)=\sum_{r=0}^{s-1} c_{l, r} I_{r, s}(t) \text { for } t \in[0, T]
$$

and $I_{r, s}$ is the indicator function of the interval $\left[t_{r}, t_{r+1}\right)$ with $t_{r}=r T / s$.

We define a metric in $P_{s}\left(J_{T}, A\right)$ by

$$
d_{P}\left(\eta_{1}, \zeta_{1}\right)=\sum_{l=1}^{m}\left\|\varphi_{l}-\psi_{l}\right\|_{L^{\infty}\left(J_{T}\right)}, \eta_{1}, \zeta_{1} \in P_{s}\left(J_{T}, A\right),
$$

where $\left\{\varphi_{l}\right\}$ and $\left\{\psi_{l}\right\}$ are the functions corresponding to $\eta_{1}$ and $\zeta_{1}$, respectively. We shall need the following lemmas, which are proved at the end of this section.

Lemma 6.3. If equation (3.1) with $\eta \in C^{\infty}\left(J_{T}, E_{1}\right)$ is uniformly $\left(\varepsilon, u_{0}, K\right)$-controllable, then there is a finite set $A=\left\{\eta_{1}^{l}, l=1, \ldots, m\right\} \subset E_{1}$, an integer $s \geq 1$ and a mapping $\Psi_{s}: K \rightarrow P_{s}\left(J_{T}, A\right)$ continuous with respect to the metric of $P_{s}\left(J_{T}, A\right)$ such that $\Psi_{s}(K) \subset \Theta\left(u_{0}, h\right)$ and

$$
\sup _{\hat{u} \in K}\left\|\mathcal{R}_{T}\left(u_{0}, \Psi_{s}(\hat{u})\right)-\hat{u}\right\|_{k}<\varepsilon .
$$

Lemma 6.4. Let $E \subset H_{\sigma}^{k+2}$ be a finite-dimensional space and $E_{1}=\mathcal{F}(E)$. Then for any $\eta_{1} \in E_{1}$ there are vectors $\zeta^{1}, \ldots, \zeta^{p}, \eta \in E$ and positive constants $\lambda_{1}, \ldots, \lambda_{p}$ whose sum is equal to 1 such that

$$
B(u)-\eta_{1}=\sum_{j=1}^{p} \lambda_{j} B\left(u+\zeta^{j}\right)-\eta \quad \text { for any } u \in H^{1} .
$$

Let $\Psi_{s}$ be the function constructed in Lemma 6.3:

$$
\Psi_{s}(\hat{u})=\sum_{l=1}^{m} \varphi_{l}(t, \hat{u}) \eta_{1}^{l}
$$

As $\eta_{1}^{l} \in E_{1}$, by Lemma 6.4 , there are vectors $\zeta^{l, 1}, \ldots, \zeta^{l, p}, \eta^{l} \in E$ and positive constants $\lambda_{l, 1}, \ldots, \lambda_{l, p}$ whose sum is equal to 1 such that

$$
B(u)-\eta_{1}^{l}=\sum_{j=1}^{p} \lambda_{l, j} B\left(u+\zeta^{l, j}\right)-\eta^{l} \quad \text { for any } u \in H^{1} .
$$

Let $u_{1}=\mathcal{R}\left(u_{0}, \Psi_{s}(\hat{u})\right)$. It follows from (6.8) that $u_{1}$ satisfies the equation

$$
\dot{u}_{1}+\sum_{j=1}^{p} \sum_{l=1}^{m} \lambda_{l, j} \varphi_{l}(t, \hat{u}) B\left(u_{1}+\zeta^{l, j}\right)=h(t)+\sum_{l=1}^{m} \varphi_{l}(t, \hat{u}) \eta^{l} .
$$

We can rewrite equation (6.9) in the form

$$
\dot{u}_{1}+\sum_{i=1}^{q} \psi_{i}(t, \hat{u}) B\left(u_{1}+\zeta^{i}\right)=h(t)+\eta(t, \hat{u}),
$$


where $\zeta^{i} \in E$ for $i=1, \ldots, q, \eta(t, \hat{u})=\sum_{l=1}^{m} \varphi_{l}(t, \hat{u}) \eta^{l}$ such that

$$
\psi_{i}(t, \hat{u})=\sum_{r=0}^{s-1} d_{i, r}(\hat{u}) I_{r, s}(t), \sum_{i=1}^{q} d_{i, r}=1
$$

for some non-negative functions $d_{i, r} \in C(K)$.

Step 2. We approximate $u_{1}$ by a solution of problem (6.1), (3.2). First we assume $s=1$. In this case (6.10) becomes

$$
\dot{u}_{1}+\sum_{i=1}^{q} d_{i}(\hat{u}) B\left(u_{1}+\zeta^{i}\right)=h(t)+\eta(\hat{u})
$$

where $d_{i} \in C(K)$ and $\eta \in C(K, E)$. Let $\zeta_{n}(t, \hat{u})=\zeta\left(\frac{n t}{T}, \hat{u}\right)$, where $\zeta(t, \hat{u})$ is a 1-periodic function such that

$$
\zeta(s, \hat{u})=\zeta^{j} \text { for } 0 \leq s-\left(d_{1}(\hat{u})+\ldots+d_{j-1}(\hat{u})\right)<d_{j}(\hat{u}), \quad j=1, \ldots, q,
$$

where $d_{0}(\hat{u})=0$. Equation (6.11) is equivalent to the equation

$$
\dot{u}_{1}+B\left(u_{1}+\zeta_{n}(t, \hat{u})\right)=h(t)+\eta(t, \hat{u})+f_{n}(t, \hat{u}),
$$

where

$$
f_{n}(t, \hat{u})=B\left(u_{1}+\zeta_{n}(t, \hat{u})\right)-\sum_{i=1}^{q} d_{i}(\hat{u}) B\left(u_{1}+\zeta^{i}\right) .
$$

Let us define

$$
\mathcal{K} g(t)=\int_{0}^{t} g(s) \mathrm{d} s
$$

Then $v_{n}=u_{1}-\mathcal{K} f_{n}$ is a solution of the problem

$$
\begin{aligned}
\dot{v}_{n}+B\left(v_{n}+\zeta_{n}(t, \hat{u})+\mathcal{K} f_{n}(t, \hat{u})\right) & =h(t)+\eta(t, \hat{u}), \\
v_{n} & =u_{0} .
\end{aligned}
$$

Suppose we have shown that

$$
\sup _{\hat{u} \in K}\left\|\mathcal{K} f_{n}(t, \hat{u})\right\|_{C\left(J_{T}, H^{k+1}\right)} \rightarrow 0 .
$$

Then $v_{n}$ satisfies

$$
\left\|v_{n}-u_{1}\right\|_{C\left(J_{T}, H^{k+1}\right)} \rightarrow 0 \text { as } n \rightarrow \infty .
$$

There is an integer $n_{0} \geq 1$ such that if $n \geq n_{0}$

$$
\sup _{\hat{u} \in K}\left\|\mathcal{R}\left(u_{0}, \zeta_{n}(\hat{u}), \eta(\hat{u})\right)-u_{1}(\cdot, \hat{u})\right\|_{C\left(J_{T}, H^{k}\right)}<\varepsilon-\hat{\varepsilon} .
$$

Then the operator

$$
\hat{\Psi}_{n}: K \rightarrow L^{1}\left(J_{T}, E\right) \times L^{2}\left(J_{T}, E\right), \quad \hat{u} \rightarrow\left(\eta(\hat{u}), \zeta_{n}(\hat{u})\right)
$$

satisfies (6.2).

To finish the proof of Proposition 6.2 in the case $s=1$, it suffices to prove (6.13). Suppose we have shown that

$$
\left\|\mathcal{K} f_{n}(t, \hat{u})\right\|_{C\left(J_{T}, H^{k+1}\right)} \rightarrow 0 \text { for any } \hat{u} \in K \text {. }
$$


To prove (6.13), by the Arzelà-Ascoli theorem, it suffices to show that the family $\left\{\hat{u} \rightarrow \mathcal{K} f_{n}(\cdot, \hat{u})\right\}$ is uniformly equicontinuous from $K$ to $C\left(J_{T}, H_{\sigma}^{k+1}\right)$. By (6.12), it suffices to show that so is $\hat{u} \rightarrow \zeta_{n}(\hat{u})$ from $K$ to $L^{1}\left(J_{T}, H_{\sigma}^{k+2}\right)$. The definition of $\zeta_{n}$ implies

$$
\begin{aligned}
\left\|\zeta_{n}\left(\cdot, \hat{u}_{1}\right)-\zeta_{n}\left(\cdot, \hat{u}_{2}\right)\right\|_{L^{2}\left(J_{T}, H^{k+2}\right)}^{2} & \leq \int_{0}^{T}\left\|\zeta\left(\frac{n t}{T}, \hat{u}_{1}\right)-\zeta\left(\frac{n t}{T}, \hat{u}_{2}\right)\right\|_{k+2}^{2} \mathrm{~d} t \\
& =\frac{T}{n} \int_{0}^{n}\left\|\zeta\left(t, \hat{u}_{1}\right)-\zeta\left(t, \hat{u}_{2}\right)\right\|_{k+2}^{2} \mathrm{~d} t \leq C \sum_{i=1}^{q}\left|d_{i}\left(\hat{u}_{1}\right)-d_{i}\left(\hat{u}_{2}\right)\right| .
\end{aligned}
$$

The uniform continuity of $d_{i}$ over $K$ gives us the required result.

Step 3. To complete the proof of Proposition 6.2 in the case $s=1$, it remains to prove (6.14). If we show that for any piecewise constant $H_{\sigma}^{k+2}$-valued function $u_{1}$ on $J_{T}$, the sequence $\left\{\mathcal{K} f_{n}\right\}$ converges to zero in the space $C\left(J_{T}, H_{\sigma}^{k+1}\right)$, then an approximation argument shows (6.14) for any $u_{1} \in C\left(J_{T}, H_{\sigma}^{k+2}\right)$.

The family $\left\{\mathcal{K} f_{n}\right\}$ is relatively compact in the space $C\left(J_{T}, H_{\sigma}^{k+1}\right)$ for any piecewise constant function $u_{1}$. Indeed, the set $f_{n}(t), t \in J_{T}$, is contained in a finite subset of $H_{\sigma}^{k+1}$ not depending on $n$. Thus, there is a compact set $G \subset H_{\sigma}^{k+1}$ such that

$$
\mathcal{K} f_{n}(t) \in G \text { for all } t \in J_{T}, n \geq 1 .
$$

As

$$
\sup _{n \geq 1}\left\|f_{n}\right\|_{C\left(J_{T}, H^{k+1}\right)}<\infty,
$$

the family $\left\{\mathcal{K} f_{n}\right\}$ is uniformly equicontinuous on $J_{T}$. Thus, by the Arzelà-Ascoli theorem, $\left\{\mathcal{K} f_{n}\right\}$ is relatively compact. Therefore convergence (6.14) will be established if we show that

$$
\mathcal{K} f_{n}(t) \rightarrow 0 \text { in } H_{\sigma}^{k+1} \text { for any } t \in J_{T} .
$$

To prove (6.15), we first assume that $u(t)=b \in H_{\sigma}^{k+2}$ for all $t \in J_{T}$. Let $t=t_{l}+\tau$, where $t_{l}=\frac{l T}{n}, l \in \mathbb{N}$ and $\tau \in\left[0, \frac{T}{n}\right)$. From the definition of $\zeta_{n}(t)$ we have

$$
\int_{0}^{\frac{l T}{n}} f_{n}(s) \mathrm{d} s=\int_{0}^{\frac{l T}{n}}\left(B\left(b+\zeta_{n}(t)\right)\right) \mathrm{d} s-\frac{l T}{n} \sum_{j=1}^{p} \lambda_{j} B\left(b+\zeta^{j}\right)=0,
$$

so

$$
\mathcal{K} f_{n}(t)=-\tau \sum_{j=1}^{p} \lambda_{j} B\left(b+\zeta^{j}\right)-\int_{0}^{\tau} B\left(b+\zeta_{n}(s)\right) \mathrm{d} s .
$$

Since $\tau \rightarrow 0$ as $n \rightarrow \infty$, we arrive at (6.15). In the same way, we can show that (6.15) holds for any piecewise constant function $u$.

The case $s \geq 2$ is deduced from the case $s=1$ exactly in the same way as in [13], Section 3.3.

Proof of Lemma 6.3. Let $\left\{e_{1}, \ldots, e_{d}\right\}$ be an orthonormal basis in $E_{1}$ with respect to scalar product $\langle\cdot, \cdot\rangle$ and $\xi_{l}(t, \hat{u}):=\left\langle\Psi_{1}(t, \hat{u}), e_{l}\right\rangle$ for $l=1, \ldots, d$. Let us define for $M=\max _{l, t, \hat{u}}\left|\xi_{l}(t, \hat{u})\right|$ and $m=2 d$ the vectors

$$
\eta_{1}^{l}=d M e_{l} \text { for } l=1, \ldots, d, \eta_{1}^{l}=-d M e_{l} \text { for } l=d+1, \ldots, m .
$$

We can see that the functions

$$
\tilde{\xi}_{l}(t, \hat{u})=\frac{1}{2 d}\left(1+\frac{\xi_{l}(t, \hat{u})}{M}\right), \tilde{\xi}_{l+d}(t, \hat{u})=\frac{1}{2 d}\left(1-\frac{\xi_{l}(t, \hat{u})}{M}\right) \text { for } l=1, \ldots, d
$$


are non-negative, their sum is equal to 1 , and they satisfy the relation

$$
\Psi_{1}(t, \hat{u})=\sum_{l=1}^{m} \tilde{\xi}_{l}(t, \hat{u}) \eta_{1}^{l}
$$

Let us define an operator $\Psi_{s}: K \rightarrow P_{s}\left(J_{T}, A\right)$ with $A=\left\{\eta_{1}^{l}, l=1, \ldots, m\right\}$ as

$$
\Psi_{s}(t, \hat{u})=\sum_{l=1}^{m} \tilde{\xi}_{l}\left(\frac{r T}{s}, \hat{u}\right) \eta_{1}^{l} \text { for } t \in\left[\frac{r T}{s}, \frac{(r+1) T}{s}\right]
$$

Since $\tilde{\xi}_{l} \in C\left(J_{T} \times K\right)$ and $K \subset H_{\sigma}^{k+2}$ is compact, we have

$$
\sup _{\hat{u} \in K}\left\|\Psi_{1}(t, \hat{u})-\Psi_{s}(t, \hat{u})\right\|_{k+2}=\sup _{\hat{u} \in K}\left\|\sum_{l=1}^{m}\left(\tilde{\xi}_{l}(t, \hat{u})-\tilde{\xi}_{l}\left(\frac{r T}{s}, \hat{u}\right)\right) \eta_{1}^{l}\right\|_{k+2} \rightarrow 0 \text { as } s \rightarrow \infty .
$$

Thus for sufficiently large $s$, we have $\Psi_{s}(K) \subset \Theta\left(h, u_{0}\right)$, and

$$
\sup _{\hat{u} \in K}\left\|\mathcal{R}_{T}\left(u_{0}, \Psi_{s}(\hat{u})\right)-\mathcal{R}_{T}\left(u_{0}, \Psi_{1}(\hat{u})\right)\right\|_{k}<\varepsilon .
$$

Hence (6.1) is uniformly $\left(\varepsilon, u_{0}, K\right)$-controllable with $\eta \in P_{s}\left(J_{T}, A\right)$.

Proof of Lemma 6.4. By the definition of $\mathcal{F}(E)$, for any $\eta_{1} \in \mathcal{F}(E)$ there are $\xi^{1}, \ldots, \xi^{n}, \eta \in E$ and positive constants $\alpha_{1}, \ldots, \alpha_{n}$ such that

$$
\eta_{1}=\eta-\sum_{i=1}^{n} \alpha_{i} B\left(\xi^{i}\right)
$$

Let us set $p=2 n, \alpha=\alpha_{1}+\ldots+\alpha_{n}$,

$$
\lambda_{i}=\lambda_{i+n}=\frac{\alpha_{i}}{2 \alpha}, \quad \zeta^{i}=-\zeta^{i+n}=\sqrt{\alpha} \xi^{i}, \quad i=1, \ldots, n
$$

Then we have

$$
B(u)-\eta_{1}=\sum_{j=1}^{p} \lambda_{j} B\left(u+\zeta^{j}\right)-\eta \quad \text { for any } u \in H_{\sigma}^{1}
$$

\section{Non CONTROLlability RESUlT}

Let us denote by $A_{T}\left(u_{0}, h, E\right)$ the set of attainability at time $T$ from $u_{0} \in H_{\sigma}^{k}$ by $E$-valued controls, i.e.,

$$
A_{T}\left(u_{0}, h, E\right)=\left\{\hat{u} \in H_{\sigma}^{k}: \hat{u}=\mathcal{R}_{T}\left(u_{0}, \eta\right) \text { for some } \eta \in \Theta\left(u_{0}, h\right)\right\}
$$

In this section, we show that the ideas of [14] can be generalized to prove that the set $A\left(u_{0}, h, E\right)=\cup_{T \in[0, \infty)}$ $A_{T}\left(u_{0}, h, E\right)$ does not contain a ball of $H_{\sigma}^{k+\gamma}, \gamma<2$ in the three-dimensional case.

Let us recall the definition of Kolmogorov $\varepsilon$-entropy (see [10]). For any $\varepsilon>0$, we denote by $N_{\varepsilon}(K)$ the minimal number of sets of diameters not exceeding $2 \varepsilon$ that are needed to cover $K$. The Kolmogorov $\varepsilon$-entropy of $K$ is defined as $H_{\varepsilon}(K)=\ln N_{\varepsilon}(K)$.

Let us consider the equation

$$
\dot{v}+B(v+z)=h .
$$


We fix an integer $k \geq 4$ and denote by $\Theta_{t}\left(h, u_{0}\right)$ the set of functions $\eta \in L^{1}\left(J_{t}, H_{\sigma}^{k}\right)$ for which (7.1), (3.2) with $z(t)=\int_{0}^{t} \eta(s) \mathrm{d} s$ has a unique solution $v \in C\left(J_{t}, H_{\sigma}^{k}\right)$. We note that

$$
\mathcal{R}_{t}\left(u_{0}, \eta\right)=v(t)+z(t)
$$

where $z(t)=\int_{0}^{t} \eta(s) \mathrm{d} s$. The following theorem is the main result of this section.

Theorem 7.1. Let $k \geq 4, u_{0} \in H_{\sigma}^{k}, h \in C\left([0, \infty), H_{\sigma}^{k}\right)$ and $E \subset H_{\sigma}^{k}$ be any finite-dimensional subspace. For any $\gamma \in[0,2)$ and any ball $Q \subset H_{\sigma}^{k+\gamma}$, we have

$$
A^{c}\left(u_{0}, h, E\right) \cap Q \neq \emptyset,
$$

where $A^{c}\left(u_{0}, h, E\right)$ is the complement of $A\left(u_{0}, h, E\right)$ in the space $H_{\sigma}^{k}$.

Proof. We argue by contradiction. Suppose that $A\left(u_{0}, h, E\right)$ contains a closed ball $Q \subset H_{\sigma}^{k+\gamma}$. Let $\left\{t_{l}\right\}$ be a dense sequence in $[0, \infty)$ and let

$$
\begin{aligned}
& D_{l, n}:=\left\{(z, y) \in W^{1,1}\left(J_{t_{l}}, H_{\sigma}^{k}\right) \cap \Theta_{t_{l}}\left(u_{0}, h\right) \times E:\|z\|_{W^{1,1}\left(J_{t_{l}}, H^{k}\right)} \leq n,\|y\|_{k} \leq n\right\}, \\
& B_{l, n}:=\left\{\hat{u} \in H_{\sigma}^{k}: \hat{u}=\mathcal{R}_{t}\left(u_{0}, z, h\right)+y \text { for some }(z, y) \in D_{l, n}, t \in\left[0, t_{l}\right]\right\} .
\end{aligned}
$$

It is easy to see that $\bigcup_{l, n} B_{l, n} \supset A\left(u_{0}, h, E\right)$. By the Baire theorem, there are integers $p$ and $m$ such that $B_{p, m}$ is dense in a ball $\hat{Q}$ with respect to the metric of $H_{\sigma}^{k+\gamma}$. Let us denote by $K:[0, \infty) \times L^{1}([0, \infty), E) \times E \rightarrow H_{\sigma}^{k-1}$ the continuous operator that takes the triple $(t, z, y) \in J_{t_{p}} \times D_{p, m}$ to $\mathcal{R}_{t}\left(u_{0}, z, h\right)+y$. As $K\left(J_{t_{p}} \times D_{p, m}\right) \subset B_{p, m}$ is closed in $H_{\sigma}^{k+\gamma} \cap B_{p, m}$, then $\hat{Q} \subset B_{p, m}$. We have from [6]

$$
H_{\varepsilon}\left(Q, L^{2}\right) \sim\left(\frac{1}{\varepsilon}\right)^{\frac{3}{k}}
$$

where $Q$ is a ball in $H^{k}$. To obtain (7.2) for any $Q \subset H_{\sigma}^{k}$, we follow the ideas of [14], Proposition 2.2. Let us denote by $\Sigma^{k}$ the closure in $H^{k}$ of the set of functions $u=\left(\partial_{2} v,-\partial_{1} v, 0\right) \in H^{k}$, where $v \in H^{k+1}$ is a scalar function. Since $\Sigma^{k}$ is closed subspace of $H_{\sigma}^{k}$, it suffices to prove (7.2) any ball $Q \subset \Sigma^{k}$. Let us introduce the set of scalar functions

$$
\dot{H}^{k}\left(\mathbb{T}^{3}\right):=\left\{u \in H^{k}\left(\mathbb{T}^{3}\right): \int_{0}^{2 \pi} u\left(x_{1}, x^{\prime}\right) \mathrm{d} x_{1}=0 \text { for any } x^{\prime} \in \mathbb{T}^{2}\right\}
$$

As

$$
H^{k}\left(\mathbb{T}^{3}\right)=\dot{H}^{k}\left(\mathbb{T}^{3}\right) \dot{+} H^{k}\left(\mathbb{T}^{2}\right),
$$

we get (7.2) for any ball $Q \subset \dot{H}^{k}\left(\mathbb{T}^{3}\right)$. Finally, if $\Pi_{2}$ is the projection $\Pi_{2}\left(u_{1}, u_{2}, u_{3}\right) \rightarrow u_{2}$, then $\Pi_{2} \Sigma^{k}=\dot{H}^{k}\left(\mathbb{T}^{3}\right)$. Thus (7.2) holds for any $Q \subset H_{\sigma}^{k}$. Hence

$$
H_{\varepsilon}\left(Q, H^{k-1}\right) \sim\left(\frac{1}{\varepsilon}\right)^{\alpha}
$$

where $Q$ is a ball in $H_{\sigma}^{k+\gamma}$ and $\alpha=\frac{3}{1+\gamma}>1$. On the other hand, from [14], (3.10), it follows that

$$
H_{\varepsilon}\left(J_{t_{p}} \times D_{p, m}, \mathbb{R} \times L^{1}(J, E) \times E\right) \prec \frac{1}{\varepsilon} \ln \frac{1}{\varepsilon} .
$$


As $h \in C\left([0, \infty), H_{\sigma}^{k}\right)$, by Theorem 2.1, the operator $K: J_{t_{p}} \times D_{p, m} \rightarrow H^{k-1}$ is Lipschitz-continuous. Then (7.4) implies

$$
H_{\varepsilon}\left(B_{p, m}, H^{k-1}\right) \prec \frac{1}{\varepsilon} \ln \frac{1}{\varepsilon} .
$$

Combining this with relation (7.3), we see that

$$
H_{\varepsilon}\left(\hat{Q}, H^{k-1}\right) \succ \varepsilon^{\nu} H_{\varepsilon}\left(B_{p, m}, H^{k-1}\right),
$$

where $\nu>0$, which contradicts the inclusion $\hat{Q} \subset B_{p, m}$.

Acknowledgements. I want to thank Armen Shirikyan for many fruitful suggestions and discussions.

\section{REFERENCES}

[1] A. Agrachev and A. Sarychev, Navier-Stokes equations controllability by means of low modes forcing. J. Math. Fluid Mech. 7 (2005) 108-152.

[2] A. Agrachev and A. Sarychev, Controllability of 2D Euler and Navier-Stokes equations by degenerate forcing. Comm. Math. Phys. 265 (2006) 673-697.

[3] J.T. Beale, T. Kato and A. Majda, Remarks on the breakdown of smooth solutions for the 3-D Euler equations. Comm. Math. Phys. 94 (1984) 61-66.

[4] P. Constantin and C. Foias, Navier-Stokes Equations. University of Chicago Press, Chicago, USA (1988).

[5] J.-M. Coron, On the controllability of 2-D incompressible perfect fluids. J. Math. Pures Appl. 75 (1996) 155-188.

[6] D.E. Edmunds and H. Triebel, Function Spaces, Entropy Numbers, Differential Operators. Cambridge University Press, Cambridge, UK (1996).

[7] E. Fernández-Cara, S. Guerrero, O.Yu. Imanuvilov and J.P. Puel, Local exact controllability of the Navier-Stokes system. J. Math. Pures Appl. 83 (2004) 1501-1542.

[8] A.V. Fursikov and O.Yu. Imanuvilov, Exact controllability of the Navier-Stokes and Boussinesq equations. Russian Math. Surveys 54 (1999) 93-146.

[9] O. Glass, Exact boundary controllability of 3-D Euler equation. ESAIM: COCV 5 (2000) 1-44.

[10] G. Lorentz, Approximation of Functions. Chelsea Publishing Co., New York, USA (1986).

[11] S.S. Rodrigues, Navier-Stokes equation on the rectangle: controllability by means of low mode forcing. J. Dyn. Control Syst. 12 (2006) 517-562.

[12] A. Shirikyan, Approximate controllability of three-dimensional Navier-Stokes equations. Comm. Math. Phys. 266 (2006) $123-151$.

[13] A. Shirikyan, Exact controllability in projections for three-dimensional Navier-Stokes equations. Ann. Inst. H. Poincaré, Anal. Non Linéaire 24 (2007) 521-537.

[14] A. Shirikyan, Euler equations are not exactly controllable by a finite-dimensional external force. Physica D 237 (2008) $1317-1323$.

[15] M.E. Taylor, Partial Differential Equations, III. Springer-Verlag, New York (1996).

[16] R. Temam, Local existence of $C^{\infty}$ solution of the Euler equation of incompressible perfect fluids. Lect. Notes Math. 565 (1976) $184-194$. 